\title{
Standard Model Higgs searches with ATLAS
}

\author{
Yaquan Fang \\ University of Wisconsin, Madison \\ On behalf of the ATLAS collaboration \\ Pheno 2011
}




\section{Introduction}

- LHC and ATLAS have successfully operated in 2010 and continue in 2011 and 2012.

- The recorded data by ATLAS detector : $45 \mathrm{pb}^{-1}$ in 2010; 267 $\mathrm{pb}^{-1}$ until May $4^{\text {th }}, 2011$.

- In this talk, we will address Higgs search with 2010 data.

$-\mathrm{H} \rightarrow \gamma \gamma$

$-\mathrm{H} \rightarrow \mathrm{WW} \rightarrow \mathrm{Ivlv} / \mathrm{lvqq}$

$-\mathrm{H} \rightarrow \mathrm{ZZ} \rightarrow \mid \mathrm{lvv} / \mathrm{llqq} / 4 \mathrm{I}$

- Is it possible that we exclude some standard model Higgs at early data?

Or what is the prospects for the exclusion/discovery in future? 


\section{SM Higgs Productions and decays}
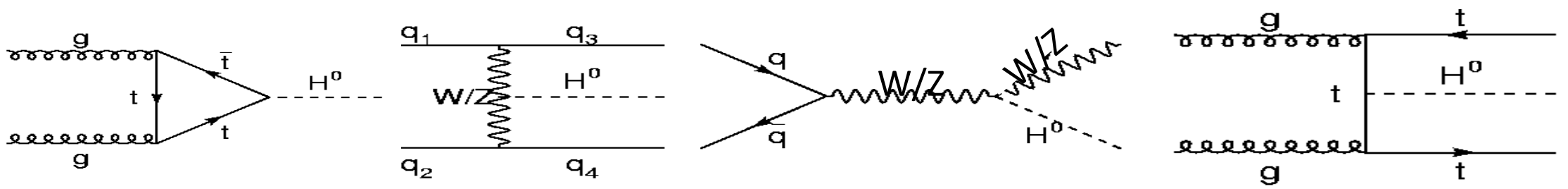

arXiv : 1101. 0593 : "Handbook of LHC Higgs Cross Sections: 1. Inclusive Observables" https://twiki.cern.ch/twiki/bin/view/LHCPhysics/CrossSections
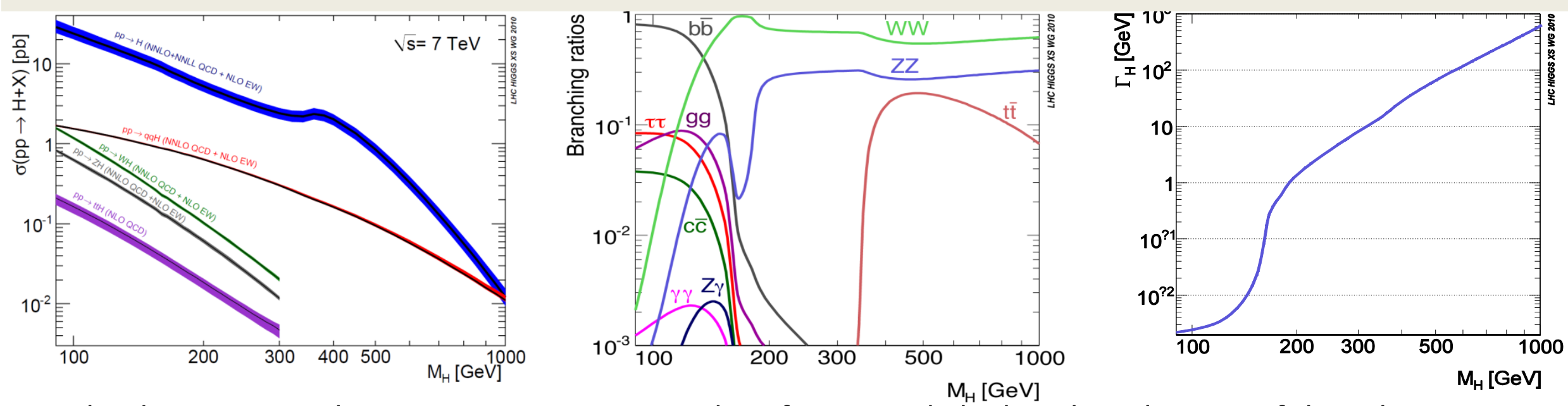

- $\quad$ The dominant production is gg Fusion: an order of magnitude higher than the sum of the other processes.

- $\quad$ VBF channel can be explored by tagging 2 forward jets and central jet veto to suppress QCD background.

- $\quad \mathrm{H} \rightarrow \gamma \gamma$ (sideband) , $\mathrm{H} \rightarrow \tau \tau$ (VBF), $\mathrm{H} \rightarrow$ bb (highest decay rate, but also high QCD background, may be feasible for the associated production) are important in low mass region.

$H \rightarrow Z Z$ : for 4 I sub-channel: clean final state, mass range $130<M_{H}<500 \mathrm{GeV}$ except $M_{H}=2 M_{w}$

for Ilvv/llqq sub-channels: higher branch ratio in $Z \rightarrow v v / q q$; on shell Z-bosons are assumed

in this study to reduce backgrounds.

- $\quad H \rightarrow W W$ : Dominant one in intermediate and high mass regions.

- Low mass region Higgs, the mass resolutions of Higgs (e.g. $H \rightarrow \gamma \gamma, H \rightarrow Z Z \rightarrow 4 I$ ) are determined by the resolution of the detector. 


\section{$\mathrm{H} \rightarrow \gamma \gamma$ channel}

ATLAS-CONF-2011-004

ATLAS-CONF-2011-025

ATLAS-CONF-2011-071 


\section{$\mathrm{H} \rightarrow \gamma \gamma$ channel with $38 \mathrm{pb}^{-1}$}

- Selection: $\mathrm{P}_{\mathrm{T} 1}>40 \mathrm{GeV}, \mathrm{P}_{\mathrm{T}_{Y 2}}>25 \mathrm{GeV}$ and $\left|\eta_{Y}\right|<2.37$ excluding the crack region.

- Relevant backgrounds are $\gamma \psi$,,$j$ jet(jet faking as photon), di-jet and small DrellYan(electron misidentified as photon):

- EM calorimeter based Photon ID and isolation efficiently suppress QCD $\psi$-jet and di-jet.

- Good reconstruction and vertex correction provide good resolution of di-photon resonance.

- The average number of reconstructed primary vertices is 2.3 with 2010 data.
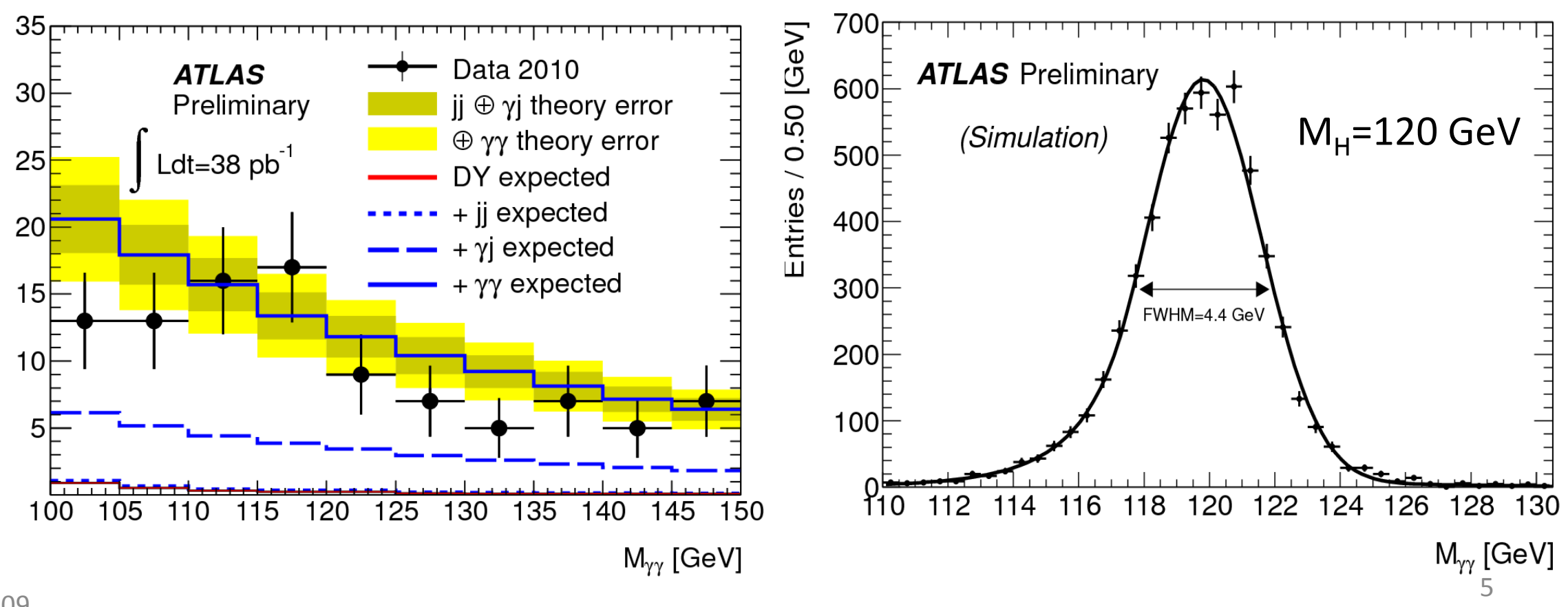


\section{Di-photon mass distribution with the combination of 2011 data}
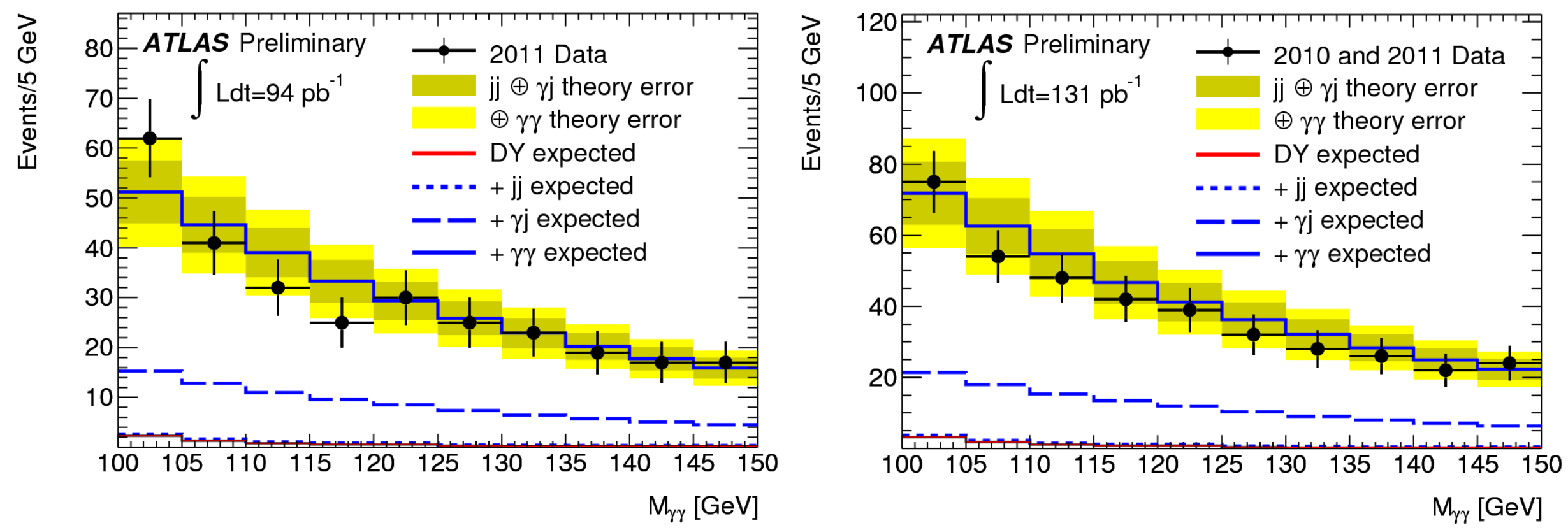

oGood agreement between data and the Standard Model prediction in the 2010+2011 analyzed data sample.

oNo excess is observed with $131 \mathrm{pb}^{-1}$ data, neither with the analysis criteria described in three notes nor with other selections studied. 
- $\quad$ Fit Model:

\section{Exclusion limit}

$\mathrm{N}_{\mathrm{s}}$ expected after selection:

- Signal described by Crystal ball plus a Gaussian.

- Background exponential (range 100-150GeV).

\begin{tabular}{cccccc}
\hline \hline Higg boson mass [GeV] & 110 & 115 & 120 & 130 & 140 \\
\hline Number of signal events & $0.43_{-0.09}^{++0.11}$ & $0.45_{-0.10}^{+0.11}$ & $0.45_{-0.10}^{+0.11}$ & $0.41_{-0.08}^{+0.10}$ & $0.31 \pm 0.08$ \\
\hline
\end{tabular}

- Background normalization and the slope of exponential are allowed to float

$$
\mathbf{N}_{\text {data }}=99
$$

- Frequentist based Profile likelihood method $\mathrm{CL}_{s+b}$ is used with Power Constrained limit (PCL):

- if the observed limit is lower than the $-1 \sigma$ of the expected limit, the later is taken as the observed limit.

- The observed limit (left) taking into account systematics is comparable to 2010 result of Tevatron.

- $\quad$ The projected limit of $1 \mathrm{fb}^{-1}$ is shown in the right plot.
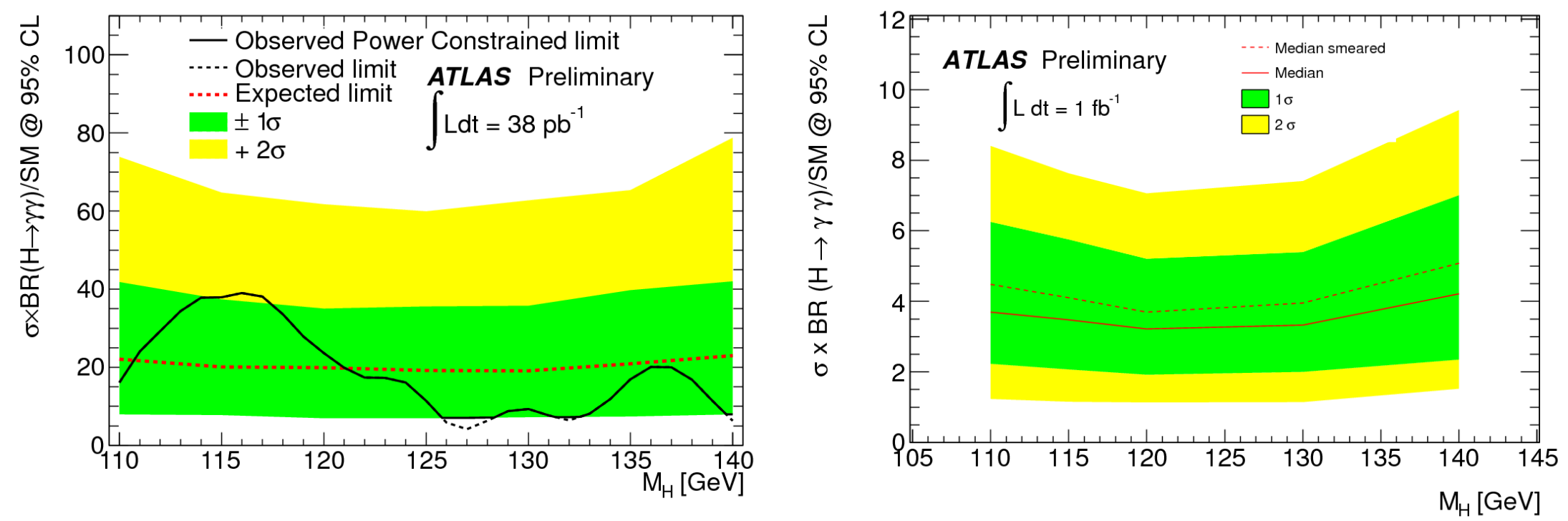


\section{$\mathrm{H} \rightarrow \mathrm{ZZ} \rightarrow 4 \mathrm{l} / \mathrm{l} q \mathrm{qq} / \mathrm{llvv}$ channel}

ATLAS-CONF-2011-026

ATLAS-CONF-2011-048 


\section{Event signature of $\mathrm{H} \rightarrow \mathrm{ZZ} \rightarrow|| \mathrm{vv} / \mathrm{Iqq} / 4 \mathrm{I}$}

- Common selection: 2 leptons $(e, m)$ with $\mathrm{P}_{\mathrm{T}}>20 \mathrm{GeV} 76<\mathrm{M}_{\|}<106 \mathrm{GeV}$

- Ilqq: final discriminant $\mathrm{M}_{\| \mathrm{ljj}} \mathrm{MET}<50 \mathrm{GeV}, 70<\mathrm{Mjj}<105 \mathrm{GeV}, \mathrm{M}_{\mathrm{H}}>360 \mathrm{GeV}: \mathrm{P}_{\mathrm{T}}(\mathrm{jets})>50 \mathrm{GeV}, \Delta \phi_{\mathrm{jj}}, \Delta \phi_{\|}<\pi / 2$

- Ilvv: final discriminant : transverse mass b-jet veto, $\mathrm{MET}>66 / 82 \mathrm{GeV}, \Delta \phi_{\|}<2.64 / 2.25$ forM $_{H}</ \geq 260 \mathrm{GeV}$

- $\quad 41: P_{\mathrm{T}}>20 \mathrm{GeV}$ for 1st/2nd lepton; $\mathrm{P}_{\mathrm{T} 3|, 4|}>15 \mathrm{GeV}(\mathrm{e}) / 7 \mathrm{GeV}(\mu) ;\left|\mathrm{M}_{|1| 2}-\mathrm{M}_{\mathrm{z}}\right|<15 / 12 \mathrm{GeV}$ for $M_{41}<170 \mathrm{GeV} />180 \mathrm{GeV}$.

- The background of subchannel llqq has higher statistics.

- Most background contributions (except ZZ) are controled by means of independent signal-free control samples.
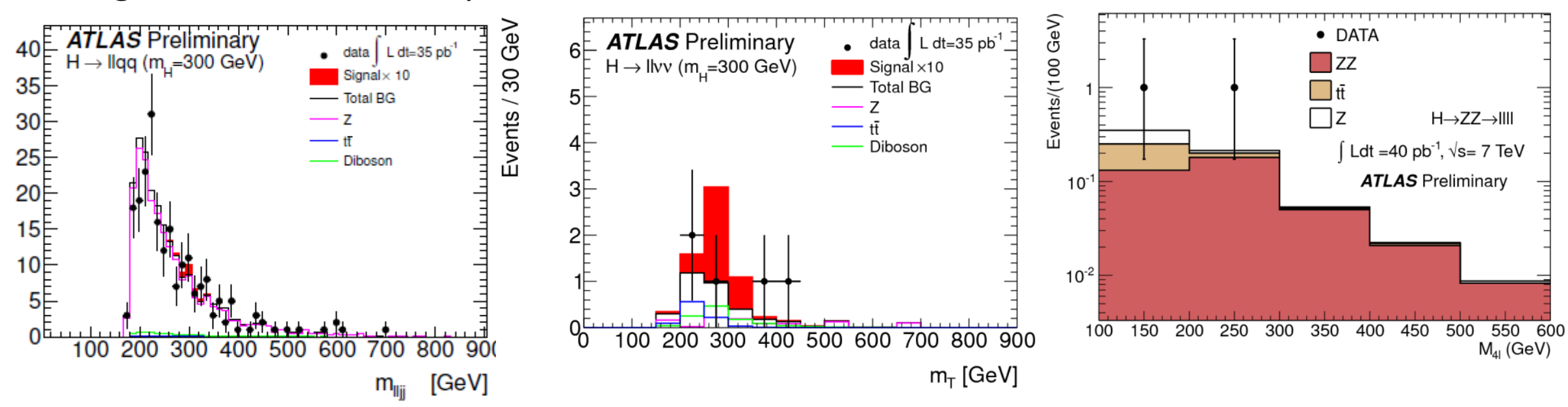

$$
m_{T}^{2} \equiv\left[\sqrt{m_{Z}^{2}+\left|\vec{p}_{T}^{\ell \ell}\right|^{2}}+\sqrt{m_{Z}^{2}+\left|\vec{p}_{T}^{\text {misss }}\right|^{2}}\right]^{2}-\left[\vec{p}_{T}^{\ell \ell}+\vec{p}_{T}^{\text {miss }}\right]^{2}
$$




\section{Limit setting for $\mathrm{H} \rightarrow \mathrm{ZZ}$}
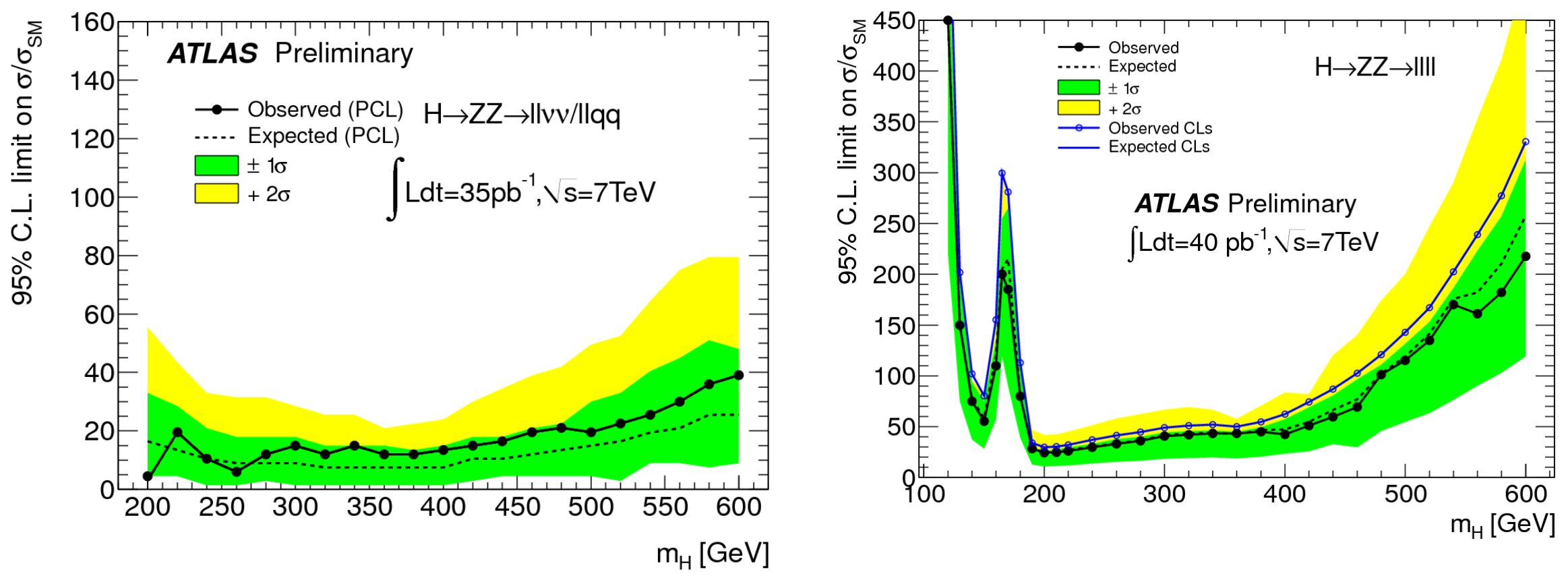

OThe observed limit for combination of $\mathrm{H} \rightarrow \mathrm{ZZ} \rightarrow \| l v \mathrm{v} /$ llqq is from 3.5 to $39 \sigma_{\mathrm{SM}}$ using $\mathrm{CL}_{\mathrm{s}+\mathrm{b}}$ method with power constrained limit. oThe lowest limit for $\mathrm{H} \rightarrow \mathrm{ZZ}^{*} \rightarrow 4 \mathrm{I}$ is 30 around $200 \mathrm{GeV}$. 
$\mathrm{H} \rightarrow \mathrm{WW} \rightarrow$ Ivlv/lvqq channel

ATLAS-CONF-2011-005

ATLAS-CONF-2011-052 


\section{The selection for $\mathrm{H} \rightarrow W W$}

- $\mathrm{H} \rightarrow \mathrm{WW} \rightarrow$ Ivlv:

- 2 leptons $(\mathrm{e}, \mu)$ with $\mathrm{P}_{\mathrm{T}}>20(15) \mathrm{GeV}$

- $M_{\|}>15,\left|M_{z}-M_{\|}\right|>10 \mathrm{GeV}$ for the same flavor leptons; $\mathrm{M}_{\|}<65 \mathrm{GeV}$

- MET>30 GeV.

- $\Delta \phi_{\|}<1.3(1.8)$ for $M_{H}<170 / \geq 170 \mathrm{GeV}$.

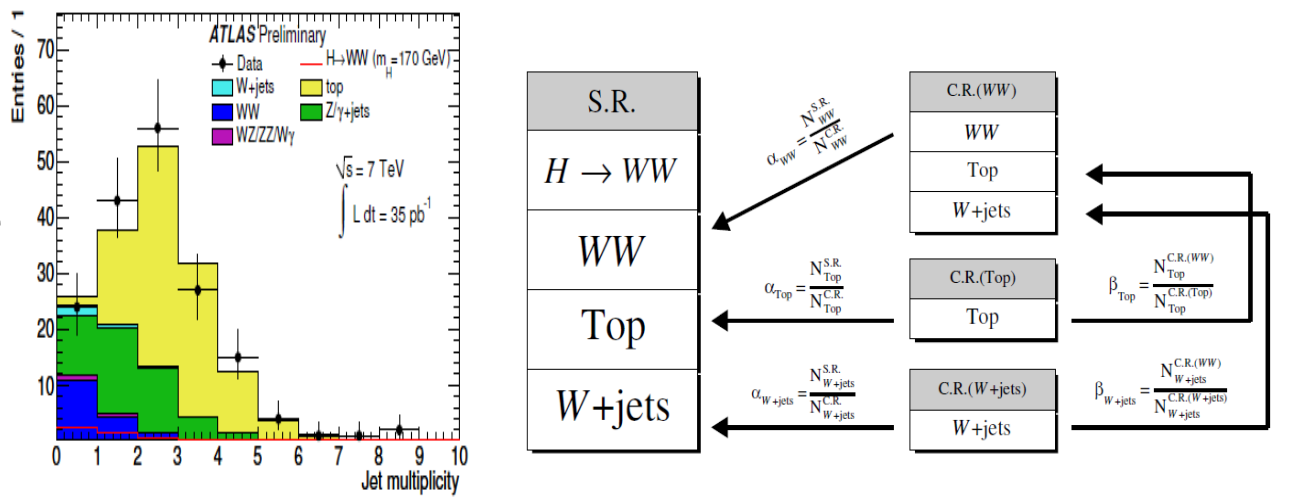

- Dedicated selections for $\mathrm{H}+0,1,2$ jets (detail in ATLAS-CONF-2011-005)

The contribution of different backgrounds are estimated with data-driven methods and various systematic uncertainties are taken into account propagating from the control region to the signal region.

- $\mathrm{H} \rightarrow \mathrm{WW} \rightarrow$ Ivqq:

- one lepton with $P_{\mathrm{T}}>30 \mathrm{GeV}$, veto events with more than one lepton with $p_{\mathrm{T}}>20 \mathrm{GeV}$.

- MET>30 GeV.

- Two /three jets with $\mathrm{P}_{\mathrm{T}}>30 \mathrm{GeV}|\eta|<4.5$, jets from W $\left(71<\mathrm{M}_{\mathrm{qq}}<91 \mathrm{GeV}\right)$ with $|\eta|<2.8 ; b$ jet veto.

- Both sub-channels categorize events with different jet bins to optimize the analysis senssitivities. 


\section{Limits for $\mathrm{H} \rightarrow \mathrm{WW} \rightarrow$ Ivlv/lvqq}
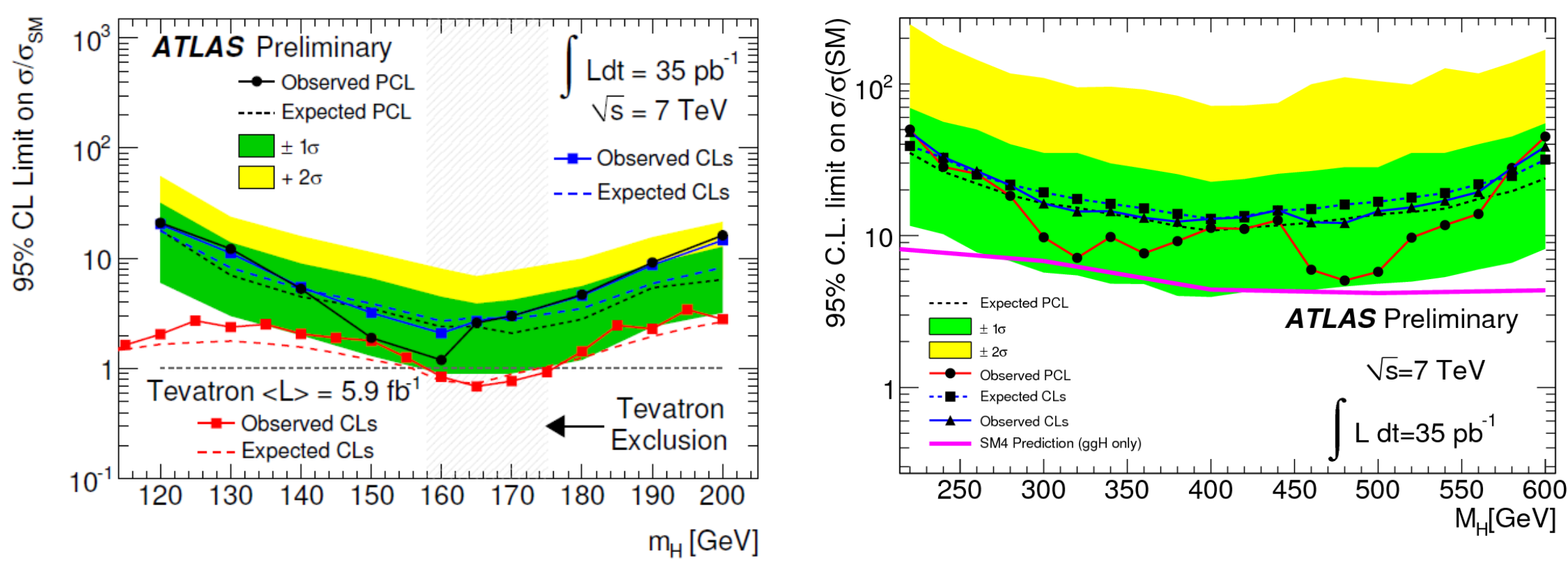

For $\mathrm{H} \rightarrow \mathrm{WW} \rightarrow$ Ivlv subchannel, $1.2 \mathrm{X} \sigma_{\mathrm{SM}}$ is excluded at $160 \mathrm{GeV}$ which is the best limit for Higgs study with 2010 data.

For $\mathrm{H} \rightarrow \mathrm{WW} \rightarrow$ Ivqq subchannel, $11.2 \mathrm{X} \sigma_{\mathrm{SM}}$ is excluded at $400 \mathrm{GeV}$. 


\section{Prospects}
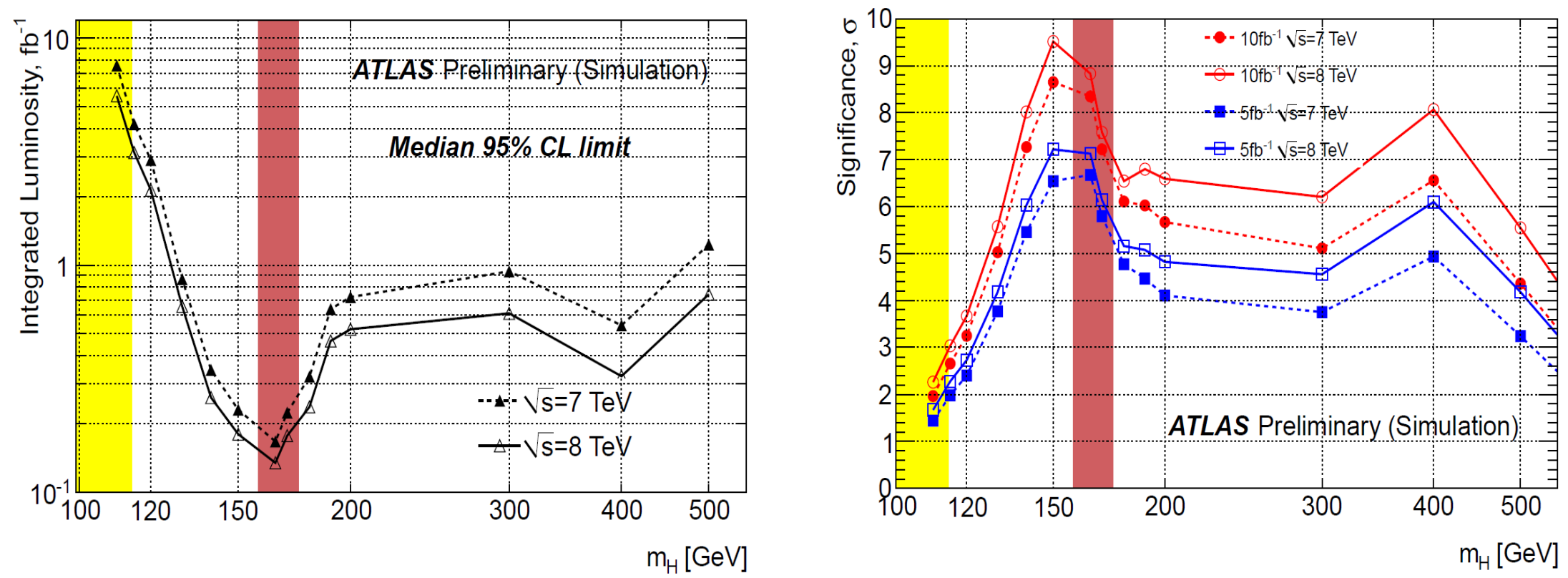

-With luminosity of a few $\mathrm{fb}^{-1}$, ATLAS expects to reach the exclusion region of LEP. -With $\sim 5 \mathrm{fb}^{-1} / 7 \mathrm{TeV}$, we start to have $5 \sigma$ discovery; we expect to have $3 \sigma$ observation in the mass range $125-500 \mathrm{GeV}$.

-With $10 \mathrm{fb}^{-1} / 7 \mathrm{TeV}$, we expect to have $5 \sigma$ discovery within mass range $130-400 \mathrm{GeV}$. 


\section{Conclusion and outlook}

- With the data of 2010, there are no SM Higgs boson mass has been excluded by the ATLAS detector.

- At $160 \mathrm{GeV}, 1.2 X \sigma_{\mathrm{SM}}$ is excluded.

- For $\mathrm{H} \rightarrow \gamma \mathrm{\gamma}$, the observed exclusion is from 8 to $38 \mathrm{SM}$ Higgs prediction. The result is comparable with the 2010 result of Tevatron.

- The region of SM Higgs boson masses above $200 \mathrm{GeV}$ has been explored for the first time.

- With a couple of $\mathrm{fb}^{-1}$, ATLAS expects to exclude the whole mass region above $115 \mathrm{GeV}$.

- With the about $5 \mathrm{fb}^{-1}$, a $5 \sigma$ discovery starts to be possible. (Of course, we prefer the latter). 


\section{Backup slides}




\section{Background Processes for $\mathrm{H} \rightarrow \gamma \gamma$}

Irreducible Bkg (diphoton):

-Born and Box processes

- Use Resbos/Diphox at NLO

- Fragmentation is taken into account at NLO by Diphox

- Difficult to simulate fragmentation, causing uncertainty $0(20 \%)$. Reducible bkg:

-Photon-jet/di-jet with one/two jet faking as a photon/photons.

-Due to excellent jet rejection, reducible bkg is comparable or smaller than irreducible bkg.

-Difficult to simulate a parton into a leading pio, which entails an uncertainty $0(50 \%)$.

Drell-Yan:

- Small contribution.

- But having single track conversions has considerably enhanced this background.
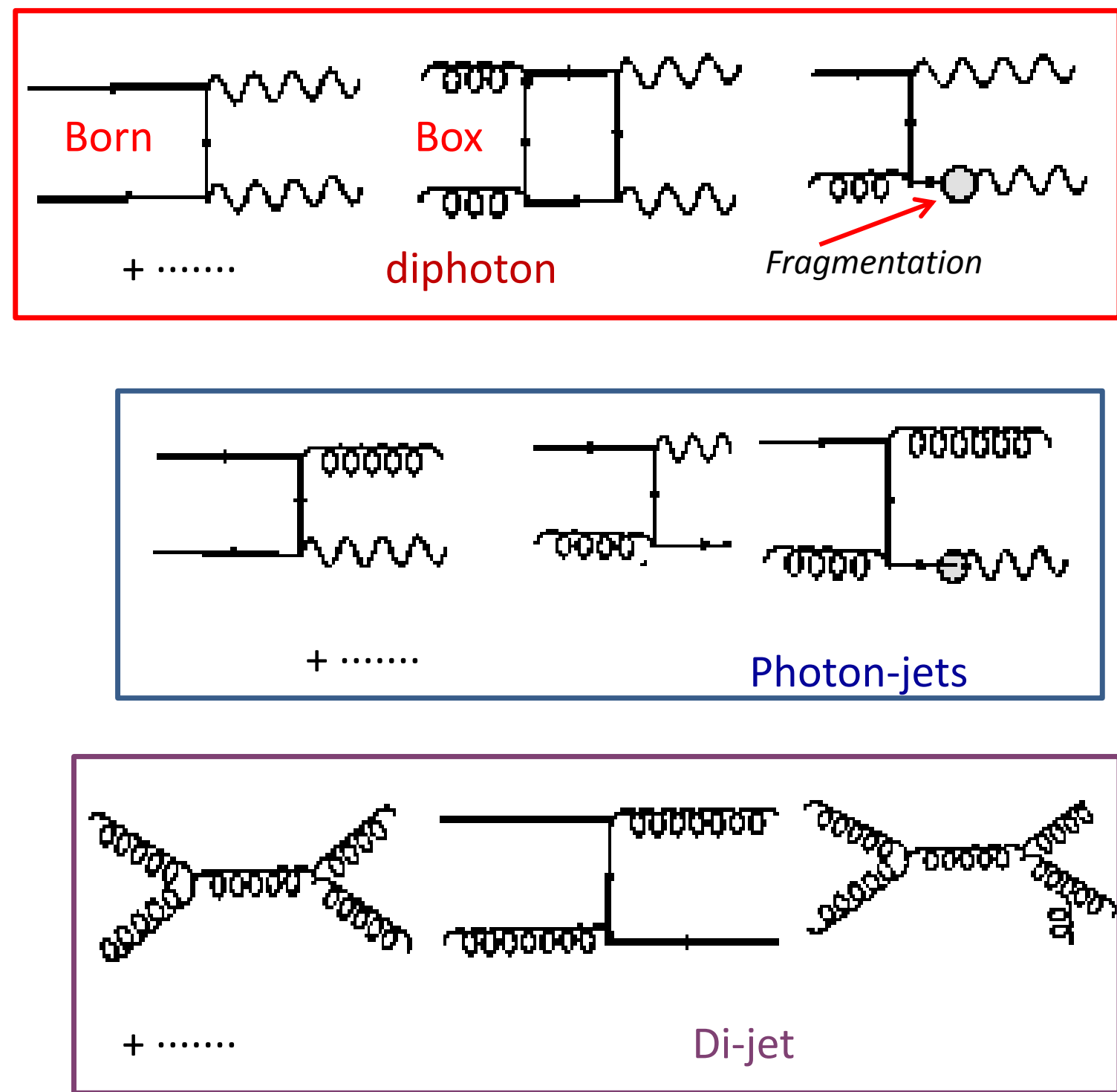


\section{$\mathrm{H} \rightarrow \gamma \gamma$ : Background estimation}
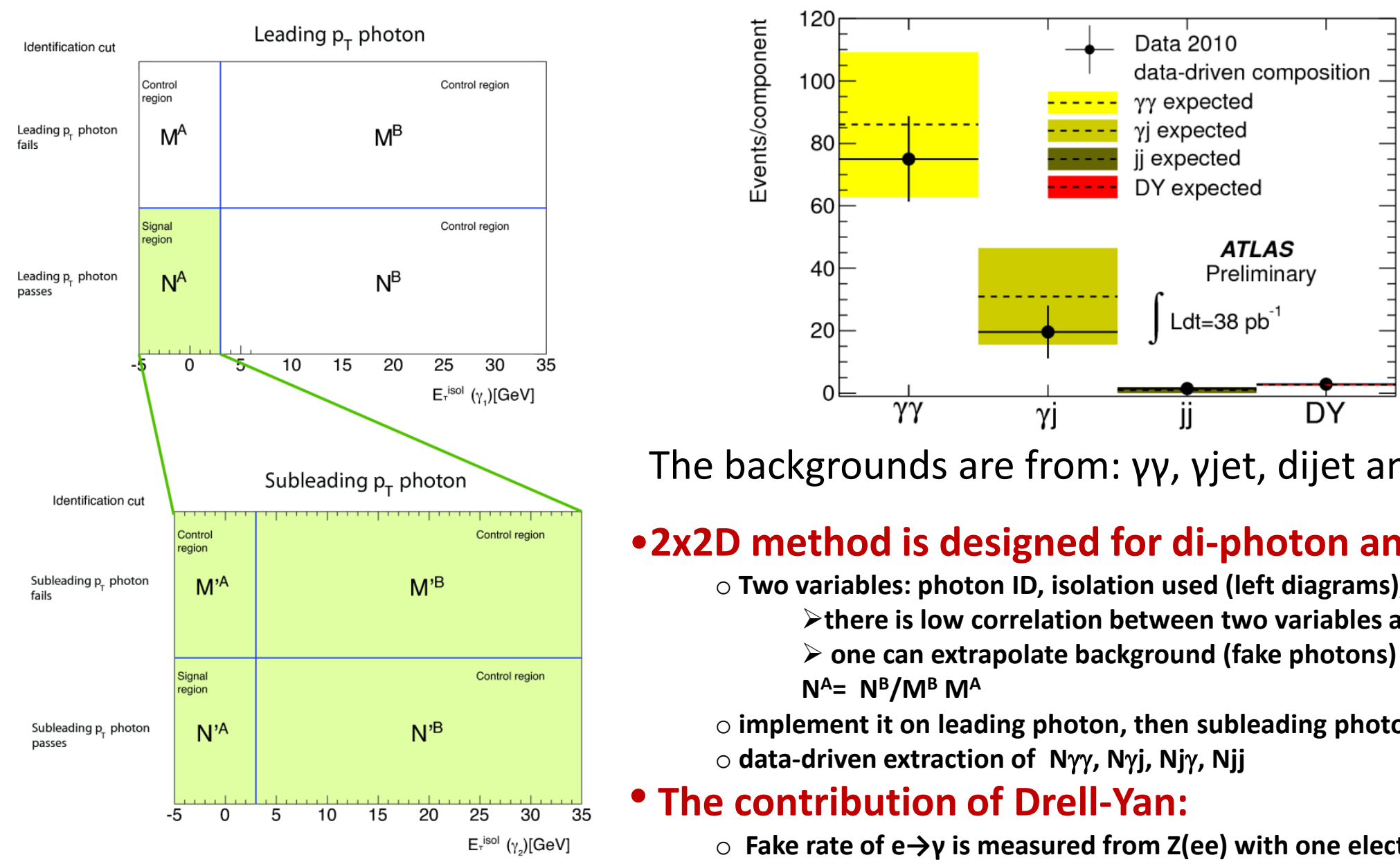

The backgrounds are from: $\gamma \psi$, vjet, dijet and Drell-Yan.

-2x2D method is designed for di-photon analysis:

○ Two variables: photon ID, isolation used (left diagrams),

$>$ there is low correlation between two variables after loose ID.

$>$ one can extrapolate background (fake photons) in signal region $N^{A}=N^{B} / M^{B} M^{A}$

$\bigcirc$ implement it on leading photon, then subleading photon (2X2D).

o data-driven extraction of $\mathrm{N} \gamma \gamma, \mathrm{N} \gamma \mathrm{j}, \mathrm{Nj} \gamma, \mathrm{N} \mathbf{j j}$

- The contribution of Drell-Yan:

$\circ$ Fake rate of $e \rightarrow \gamma$ is measured from $Z(e e)$ with one electron faking as a photon

- The estimated components of backgrounds are consistent with theoretical prediction within uncertainties. 


\section{Extraction of Exclusion Sensitivity}

- We perform a statistical study of the exclusion sensitivity at $38 \mathrm{pb}^{-1}$.

a: $\quad C L s+b$ method: $C L=1-P_{\text {value }}(s+b)$ with test statistic :

$$
\tilde{q}_{\mu}= \begin{cases}-2 \ln \frac{\mathcal{L}(\mu, \hat{\boldsymbol{\theta}})}{\mathcal{L}(0, \hat{\boldsymbol{\Theta}})} & \hat{\mu}<0, \\ -2 \ln \frac{\mathcal{L}(\mu, \hat{\boldsymbol{\theta}})}{\mathcal{L}(\hat{\mu}, \hat{\boldsymbol{\theta}})} & 0 \leq \hat{\mu} \leq \mu, \\ 0 & \hat{\mu}>\mu\end{cases}
$$

Power constraint limit (the observed limit is lower than the $-1 \sigma$ of the expected limit, the later is taken as the observed limit) is used for the observed limit to reduce false exclusion.

Background: shape and normalization are the nuisance parameters.

b: $\mathrm{CL}_{\mathrm{s}}$ is used for the purpose of comparison with results from Tevatron. reduce the false exclusion, but conservative. 


\section{Systematics in $\mathrm{H} \rightarrow \gamma p$}

\begin{tabular}{rl|c}
\hline \hline & Source & Uncertainty \\
\hline & Luminosity & $\pm 3.4 \%$ \\
\hline Theory & Cross-section (scales) & $+20 \%$ \\
\hline Efficiency & Photon identification & $\pm 11 \%$ \\
& Photon isolation & $\pm 10 \%$ \\
& Trigger & $+1.1 \%$ \\
\hline Resolution & Calibration & \\
& $e \rightarrow \gamma$ extrapolation & $\pm 13 \%$ \\
& Pile-up & \\
\hline
\end{tabular}




\section{CLs limit for $H \rightarrow \gamma \gamma$}

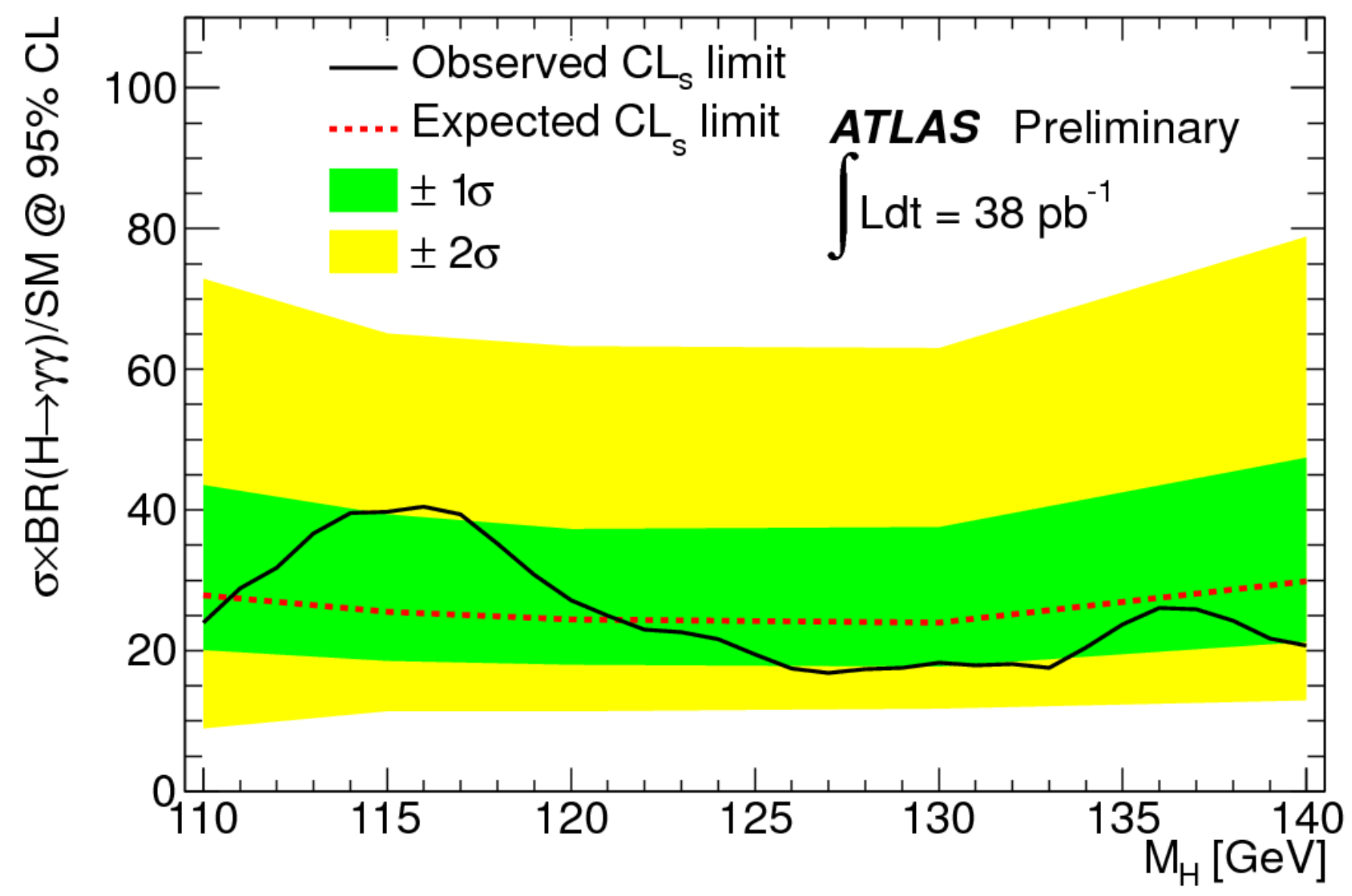




\section{Limits for $\mathrm{H} \rightarrow \mathrm{ZZ} \rightarrow \mid \mathrm{lvv} / \mathrm{Ilqq}$}
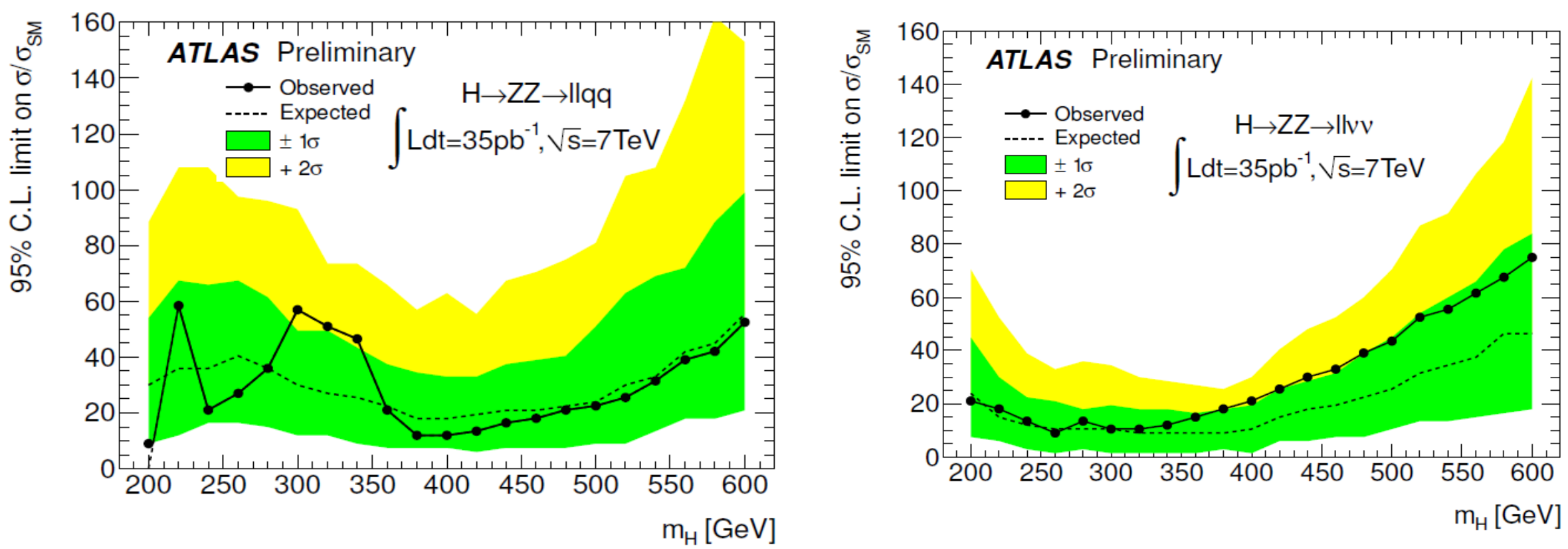